\title{
A RELAÇÃO DA QUALIDADE DE VIDA ENTRE RESPIRADORES BUCAIS, ORONASAIS E NASAIS
}

Beatriz Cristina Bincoleto, Kemelli Adriana P.B. de Almeida, Sandra Silva Lustosa, Meira Aparecida Judai.

Universidade do Oeste Paulista - UNOESTE, Curso de Fonoaudiologia - Presidente Prudente (SP), Brasil. E-mail: beatriz_cristina.96@hotmail.com; sandralustosa@outlook.com

\section{RESUMO}

O presente estudo verificou a relação entre a qualidade de vida e o padrão respiratório e a influência deste no bem-estar físico, emocional e social dos indivíduos que apresentam respiração oral, oronasal e nasal. Foram investigados 30 participantes, de ambos os sexos, sendo 15 respiradores orais e oronasais, 15 respiradores nasais, entre 13 a 60 anos. Foi utilizado um protocolo de Qualidade de Vida validado - o World Health Organization Quality Life - WHOQOLBREF (WHOQ) versão em português. Houve associação entre os grupos estudados nos domínios relações físicas e sociais, com valores $(p<0,05)$ arbitrados, onde média dos escores entre respiradores orais, oronasais em relação aos nasais, mostrando que o respirador nasal tem melhor qualidade de vida entre esses domínios. O estudo revelou que respiradores orais e oronasais tem pior qualidade de vida que respiradores nasais nos aspectos físico e social.

Palavras-chave: Respiração, qualidade de vida, respiração bucal, fonoaudiologia.

\section{THE RELATIONSHIP OF THE QUALITY OF LIFE OF ORAL, ORONASAL AND NASAL RESPIRATORS}

\begin{abstract}
The present study aimed to verify the relationship between quality of life and respiratory its influence on the physical, emotional and social well-being of individuals who present oral, oronasal and nasal breathing. 30 participants, of both sexes, were investigated: 15 oral and oronasal breathers, 15 nasal breathers, between 13 and 60 years old. A valid quality of life protocol - the World Health Organization quality Life - WHOQOL-BREF (WHOQ) Portuguese version was used. the existence of association between the groups studied in social and physical domains, with values $(p<0.05)$ arbitrated, where mean of oral or nasal breath scores, was nasal respirator has better quality of life between domains. The study revealed that oral and oronasal breathers have worse quality of life than nasal breathers in physical and social aspects.
\end{abstract}

Key words: Respiration, quality of life, mouth Breathing, phonoaudiology. 


\section{INTRODUÇÃO}

A qualidade de vida tornou-se um conceito amplamente discutido e vem sendo debatido atualmente dentro da área da saúde. Em 1948, a Organização Mundial de Saúde (OMS), definiu saúde como não apenas a ausência de doença ou enfermidade, mas também a presença de bemestar físico, mental e social. Recentemente este conceito foi modificado pela OMS para "[...] a percepção do indivíduo sobre a sua posição na vida, no contexto da cultura e dos sistemas de valores nos quais ele vive, e em relação a seus objetivos, expectativas, padrões e preocupações" (1).

A qualidade de vida influência na respiração e em seus domínios físico, psicológico, emocional e ambiental tanto na fase infantil como na adulta, sendo que na infância pode influenciar no desenvolvimento escolar, bem-estar físico e social. Já na fase adulta, observa-se que o indivíduo com uma má qualidade de vida apresentará sentimentos negativos, mau humor, ansiedade e na maioria das vezes não estará satisfeito com sua aparência, vida sexual, não conseguindo realizar suas atividades do dia-a-dia, tendo dificuldades no seu desenvolvimento pessoal, no trabalho e consigo mesmo.

O indivíduo com a respiração oral pode ter problemas de comportamento tais como: hiperatividade, distúrbios de atenção, comportamento antissocial, interferência emocional e agressividade, podendo vir adquirir o distúrbio de aprendizagem, devido à dificuldade de se concentrar em uma atividade por ter sua atenção seletiva prejudicada. A etiologia da respiração oral é, muitas vezes multifatorial, podendo causar alteração no processamento das informações auditivas, dificuldades de relacionamento interpessoal, déficit de atenção e cognição, o que poderá acarretar danos ao processo de alfabetização e, consequentemente, à aquisição da linguagem escrita ${ }^{(2)}$.

Para discutir possíveis direcionamentos para a relação da qualidade de vida e padrão respiratório é interessante analisar, ainda que brevemente, o processo da respiração oral, oranasal, e nasal.

Uma respiração ineficiente, quando realizada exclusivamente pela boca, pode ser considerada como uma adaptação patológica, em virtude da dificuldade de se respirar pelo nariz, favorecendo a inspiração de ar mais seco não filtrado podendo provocar processos inflamatórios crônicos ${ }^{(3)}$. Tal adaptação acarretará na hipotonia dos músculos faciais, tônus da língua, lábios, orbicular da boca, bucinador, e também podendo ter dores na ATM, problemas oclusais, disfonias e apneia do sono ${ }^{(4)}$.

Perante isso, o Grupo de Qualidade de Vida da OMS desenvolveu uma medida de Qualidade de Vida aplicável para uso em diversas culturas, cujo resultado se deu pela elaboração do World Health Organization Quality of Life-100 (WHOQOL-100) ${ }^{(12)}$.

A partir do WHOQOL-100, foi desenvolvido O WHOQOL-bref devido à necessidade de instrumentos curtos e de rápida aplicação, podendo ser utilizado tanto para populações que contém algum tipo de doença como em populações saudáveis. Por meio da utilização do WHOQOL-bref, podem ser gerados novos conhecimentos, ser levantado questionamentos contribuir para a tomada de decisões que melhorem de fato a qualidade de vida das pessoas ${ }^{(5)}$.

Com este estudo, buscou-se verificar a qualidade de vida e o padrão respiratório, deve-se ao fato da causa de alterações em vários órgãos e sistemas estando ligados não só à capacidade vital do individuo, mas também à influência direta na qualidade de vida, tais como: a influência deste no bemestar físico, emocional e social, de pessoas que apresentem a respiração oral e oro nasal, em comparação com pessoas que apresentem a respiração nasal. 


\section{MATERIAL E MÉTODOS}

O presente estudo foi enviado para a apreciação do comitê de ética em pesquisa (CEP) e Comitê Assessor de Pesquisa Institucional (CAPI), da Universidade do Oeste Paulista de Presidente Prudente - SP, e aprovado pelo CEP protocolo no 80729117.4.0000.5515 e CAPI protocolo n 4278 .

Trata-se de um estudo de campo, de caráter transversal, composto de 30 participantes, de ambos os sexos, sendo 15 respiradores orais e/ou oronasais (10 do sexo masculino e 5 do sexo feminino), e 15 respiradores nasais (5 do sexo masculino e 10 do sexo feminino), com faixa etária entre 13 a 60 anos. A amplitude da faixa etária justifica-se por a coleta ter sido realizada em uma clínica Escola de Fonoaudiologia onde o número de pacientes é restrito.

Os critérios de inclusão utilizados foram sujeitos que respiram parcialmente ou integralmente pela boca e respiradores nasais, e os de exclusão foram os que apresentarem alterações neurológicas, síndrome genéticas diagnosticadas, depressão, compulsão, deformidades craniofaciais, e/ou os que apresentaram histórico crônico de comprometimento emocional como ansiedade.

O convite para fazer parte da amostra foi realizado com a apresentação do Termo de Consentimento Livre e Esclarecido - TCLE, mediante assinatura do participante, foi explicado sobre o funcionamento da pesquisa e as orientações sobre a realização da mesma. O instrumento de coleta foi um protocolo de Qualidade de Vida validado - o World Health Organization Quality Life - WHOQOL-BREF (WHOQ), composto por 24 questões divididas nos domínios, físico, psicológico, relações social e meio ambiente, além de duas questões gerais. Nas perguntas referentes ao domínio físico foram questionados sobre dor, desconforto, energia, fadiga, mobilidade, atividades da vida cotidiana, dependência de medicação ou de tratamentos e capacidade de trabalho. No domínio psicológico as questões foram relativa à autoestima, aparência, sentimento negativo e positivo e religião. No que se refere às questões da relação social foram explorados as relações pessoais, apoio social e atividade sexual. E no domínio ambiental, segurança física e proteção, ambiente no lar e vida social, recursos financeiros, cuidados de saúde, ambiente físico e transporte.

As comparações entre os tipos de respiradores (oral/oronasal e nasal) foram realizadas utilizando-se o teste $t$ de Student e as associações entre os domínios e as faixas etárias foram realizadas utilizando-se o teste Kruskal Wallis e em caso de diferença significativa as médias foram comparadas pelo teste de Dunn. As análises estatísticas foram realizadas no programa BioEstat 5.3 utilizando nível de significância de $5 \%(p<0,05)$.

\section{RESULTADOS}

$\mathrm{Na}$ Tabela 1, mostra os indicadores de qualidade de vida entre os grupos de respiradores orais/oronasais e nasais, estão apresentados os resultados referentes à faixa etária. 
Tabela 1. Valores médios dos domínios do protocolo WHOQOL-BREF (WHOQ) dos respiradores orais e oronasais e nasais de acordo com a faixa etária.

\begin{tabular}{|c|c|c|}
\hline \multirow{2}{*}{ Faixa etária } & Orais e oronasais & Nasais \\
\hline & \multicolumn{2}{|c|}{ Percepção da qualidade de vida } \\
\hline 13 a 20 & 4.20 & 4.25 \\
\hline 21 a 30 & 3.00 & 2.50 \\
\hline 31 a 40 & 4.00 & 4.00 \\
\hline 41 a 50 & 4.00 & 4.50 \\
\hline 51 a 60 & - & 3.00 \\
\hline \multirow[t]{2}{*}{ Valor de $p$} & 0.1129 & 0.1540 \\
\hline & \multicolumn{2}{|c|}{ Satisfação com a saúde } \\
\hline 13 a 20 & 3.80 & 4.00 \\
\hline 21 a 30 & 3.00 & 3.00 \\
\hline 31 a 40 & 4.00 & 4.00 \\
\hline 41 a 50 & 4.00 & 4.00 \\
\hline 51 a 60 & - & 2.00 \\
\hline \multirow[t]{2}{*}{ Valor de $p$} & 0.2989 & 0.2635 \\
\hline & \multicolumn{2}{|c|}{ Domínio físico } \\
\hline 13 a 20 & 3.11 & 3.32 \\
\hline 21 a 30 & 3.19 & 2.71 \\
\hline 31 a 40 & 3.48 & 3.57 \\
\hline 41 a 50 & 3.29 & 3.71 \\
\hline 51 a 60 & - & 3.00 \\
\hline \multirow[t]{2}{*}{ Valor de $p$} & 0.7594 & 0.1361 \\
\hline & \multicolumn{2}{|c|}{ Domínio psicológico } \\
\hline 13 a 20 & $3.50 a b$ & 3.56 \\
\hline 21 a 30 & $3.02 \mathrm{~b}$ & 3.17 \\
\hline 31 a 40 & $3.89 \mathrm{a}$ & 3.50 \\
\hline 41 a 50 & $3.33 a b$ & 3.91 \\
\hline 51 a 60 & - & 2.83 \\
\hline \multirow[t]{2}{*}{ Valor de $p$} & 0.0416 & 0.2443 \\
\hline & \multicolumn{2}{|c|}{ Relações sociais } \\
\hline 13 a 20 & 3.47 & 4.00 \\
\hline 21 a 30 & 3.17 & 3.00 \\
\hline 31 a 40 & 3.33 & 4.33 \\
\hline 41 a 50 & 4.00 & 4.50 \\
\hline 51 a 60 & - & 3.67 \\
\hline \multirow[t]{2}{*}{ Valor de $p$} & 0.6160 & 0.1688 \\
\hline & \multicolumn{2}{|c|}{ Meio ambiente } \\
\hline 13 a 20 & 3.63 & $3.94 \mathrm{a}$ \\
\hline 21 a 30 & 3.21 & $2.69 \mathrm{~b}$ \\
\hline 31 a 40 & 3.75 & $3.81 \mathrm{ab}$ \\
\hline 41 a 50 & 3.25 & $3.88 a b$ \\
\hline 51 a 60 & - & $3.38 \mathrm{ab}$ \\
\hline Valor de $p$ & 0.0563 & 0.0164 \\
\hline
\end{tabular}

Os resultados estatísticos demonstraram existência de associação entre os grupos estudados nos domínios em relações físicas e sociais, com valores de $p<0,05$ arbitrado, onde a 
média dos escores entre respiradores orais e oronasais com relação aos nasais mostrando que o respirador nasal tem melhor qualidade de vida entre esses domínios. Somente não foram encontrados indícios de associação no nível de significância de 5\% nos domínios psicológico, ambiental e nas questões sobre percepção da qualidade de vida e satisfação com a saúde entre os três grupos. Em relação à média dos escores entre a qualidade de vida, os resultados demonstram que: mesmo com a alteração na respiração entre esses domínios a qualidade de vida foi à mesma, exceto no grupo dos respiradores orais / oronasais no domínio físico e social.

\section{DISCUSSÃO E CONCLUSÃO}

O conceito preconizado pela Organização Mundial da Saúde (OMS) refere que a qualidade de vida reflete a percepção dos indivíduos de que suas necessidades estão sendo satisfeitas ou, ainda, que lhes estão sendo negadas oportunidades de alcançar a felicidade e a auto-realização, com independência de seu estado de saúde físico ou das condições sociais e econômicas (OMS, 1998).

Há inúmeras evidências que demonstram a contribuição do estudo da qualidade de vida para a saúde ${ }^{(14)}$, seu estudo vai além da contribuição dos componentes da vida social gerando conhecimentos sobre os sentimentos e comportamento das pessoas no seu dia-a-dia, ampliando dessa forma as ações fonoaudiológicas e interdisciplinares no âmbito da promoção da saúde ${ }^{(11)}$.

Os indivíduos que apresentam ineficiência respiratória podem apresentar hipotonia dos músculos faciais, tônus da língua, lábios, orbicular da boca e bucinador, também podendo ter dores na ATM, problemas oclusais, disfonias e apneia do sono ${ }^{(5)}$. A literatura ${ }^{(6)}$ evidencia que sujeitos com tais alterações, seriam afetados em alguns aspectos a sua qualidade de vida.

No presente estudo verificou se há associação quanto à qualidade de vida de sujeitos com ineficiência respiratória em relação aos participantes sem alterações na respiração. Os respiradores nasais não apresentaram diferenças em relação aos respiradores oronasais/nasais nas questões de percepção da qualidade de vida, satisfação com a saúde, domínio psicológico e meio ambiente. Acredita-se que diante do reconhecimento das condições físicas e domínio psicológico pode ser também afetado, por alterações na concentração, humor, rendimento, aparência física e ansiedade. Estudos na área da odontologia por ${ }^{(7)}$, evidenciam uma pior qualidade de vida quanto aos respiradores orais e oronasais, onde os mesmos tem uma dificuldade na respiração por apresentarem um mecanismo compensatório, diante de uma respiração nasal ineficiente.

As dificuldades respiratórias apresentadas pelos respiradores orais e oronasais podem ser inúmeras sendo obtida de forma isolada, como eversão de lábio, sonolência diurna, falta de atenção, baixa concentração dentre outras. Já no quesito das alterações comportamentais constatou-se na literatura ${ }^{(8)}$ que os respiradores orais apresentam com frequência sinais de irritação, mau humor, inquietude, desatenção, agitação, ansiedade, medo, depressão, desconfiança, déficit de aprendizagem e impulsividade.

Pudemos observar neste estudo, que as relações interpessoais e qualidade de vida estão diretamente interligadas, sendo a percepção dos indivíduos primordial, pois através dela ocorre o possível autojulgamento perante os aspectos comportamentais, aceitação social, competências escolares e aparência física.

$\mathrm{Na}$ análise e comparação da qualidade de vida entre os respiradores orais, oro nasais e nasais foi detectada uma diferença significante entre os grupos, sendo nos domínios físico e social no grupo de respiradores orais/oronasais de acordo com a faixa etária analisados pelo WHOQOLBREF exceto nas questões de percepção da qualidade de vida, satisfação com a saúde, domínio psicológico e meio ambiente.

Evidenciou-se com isso um parâmetro na qualidade de vida dos respiradores nasais sendo equivalente aos respiradores orais e oro nasais, que na maior parte das vezes, possuem resultados 
de uma qualidade de vida inferior pelo fato de caracterizar alterações mecânicas das vias aéreas superiores e/ou alterações faciais. Outros fatores que podemos constatar que vem a influenciar são os demográficos (idade, gênero, e estatuto socioeconômico) e as características intrapessoais/ psicossociais (autoconceito), pois ao longo da idade já se consegue fazer julgamentos do seu valor e seu autojulgamento perante as competências escolares, aceitação social, aparência física e aspectos comportamentais, que corrobora para a hipótese fundamental do estudo de que os grupos apresentam diferenças significantes na qualidade de vida.

A partir dos resultados obtidos, observou-se a relevância em investigar na população os sujeitos com ineficiência respiratória, visto que a maior parte dos respiradores orais, oronasais não tem a ciência quanto a sua alteração e a percepção em relação à qualidade de vida. $O$ estudo revelou que respiradores orais e oro nasais tem pior qualidade de vida que respiradores nasais nos aspectos físico e social.

\section{REFERÊNCIAS}

1. Kluthcovsky AC GC, Kluthcovsky FA. O WHOQOL-bref, um instrumento para avaliar qualidade de vida: uma revisão sistemática. Rev. psiquiatr. Rio Gd. Sul. 2009; 31 https://doi.org/10.1590/S0101-81082009000400007

2. Chedid KA, Kuhn RC, Di Francesco, and Paula Andreya de Souza Junqueira. A INFLUÊNCIA DA RESPIRAÇÃO ORAL NO PROCESSO DE APRENDIZAGEM DA LEITURA E ESCRITA EM CRIANÇAS PRÉESCOLARES.

4. Marchesan IQ. A equipe de trabalho no respirador oral. In: Krakauer LH, Di Francesco RC, Marchesan IQ, organizadoras. Conhecimentos essenciais para entender bem a respiração oral. São José dos Campos: Pulso. p.163-167, 2003.

5. Rizzo MC. O respirador bucal. In: Naspitz C. Alergias respiratórias. São Paulo: Vivali Ed. 1, p.98110, 2003.

6. Campanha SMA, Freire LMS, Fontes MF. O impacto da asma, da rinite alérgica e da respiração oral na qualidade de vida de crianças e adolescentes. Rev. CEFAC. 2008; 10(4): 513-519. https://doi.org/10.1590/S1516-18462008000400011

7. Menezes VA, Tavares RLO, Garcia AFG. Síndrome da respiração oral: alterações clínicas e comportamentais. Arquivos em Odontologia. 2009; 45(3): 160-5.

8. Campanha SMA, Freire LMS, Fontes MJF. O impacto da asma, da rinite alérgica e da respiração oral na qualidade de vida de crianças e adolescentes. Rev. CEFAC. 2008;10(4):513-9. https://doi.org/10.1590/S1516-18462008000400011

9. Minayo MCS, Hartz ZMA, Buss PM. Qualidade de vida e saúde: um debate necessário. Ciênc. Saúde coletiva. 2000; 5(1): 7-18. https://doi.org/10.1590/S1413-81232000000100002

10. PREBIANCHI, HB. Medidas de qualidade de vida para crianças: aspectos conceituais e metodológicos. Psicol. teor. prat., São Paulo, v. 5, n. 1, p. 57-69, jun. 2003. [Acesso em: 18 maio. 2017]. Disponível em: <www.scielo.br>.

11. Campos, MO; Rodrigues Neto. JF. Qualidade de vida: um instrumento para promoção de saúde. Revista Baiana de saúde pública v. 32, n. 2, p.232, 2014. 
12. Fleck MPA, Louzada S, Xavier M, Chachamovich E, Vieira G, Santos L et al. Aplicação da versão em português do instrumento abreviado de avaliação da qualidade de vida "WHOQOL-bref". Rev. Saúde Pública. 2000 Apr; 34(2): 178-183. https://doi.org/10.1590/S0034-89102000000200012

13. Marchesan, IQ. AVALIAÇÃO E TERAPIA DOS PROBLEMAS DA RESPIRAÇÃO.

14. Seidl EMF, Zannon CMLC. Qualidade de vida e saúde: aspectos conceituais e metodológicos. Cad. Saúde Pública. 2004 Apr; 20(2): 580-588. https://doi.org/10.1590/S0102$\underline{311 \times 2004000200027}$ 